\title{
Utility of MRI in diagnosis of molecular subtypes of breast cancer
}

\section{Ebtsam Ahmed Mohammed ${ }^{a}$, Mohammad Tharwat Mahmoud Solymana, Nagham Nabil Omar $^{b}$, Nahla Mohamed Ali Hasan ${ }^{a}$}

a Department of Radio-Diagnosis, Faculty of Medicine, Sohag University, Sohag, Egypt.

${ }^{b}$ Department of Radio-Diagnosis, Faculty of Medicine, Assiut University, Assiut, Egypt.

\section{Abstract}

Background: Breast cancer is a collection of diseases defined by distinct pathological (e.g., ductal, lobular, mucinous) and molecular characteristics (e.g., ER and PR, HER2 amplification, and more recently transcriptome-based classifications such as luminal and basal cancers). Molecular subtyping is beneficial for the diagnosis and individualized treatment of breast cancer.MRI is a supplemental technique to mammography and ultrasonography for the evaluation of breast lesions and to predict molecular subtypes of breast cancer. Studies have highlighted the value of DCE-MRI in reflecting the anatomic and functional properties of tumors and facilitating treatment.

Objectives: The aim of this work was to assess the utility of MRI as an accurate method for detection of molecular subtypes of breast cancer.

Patients and Methods: a retrospective clinical study of 2-years enrollment duration. The study was conducted at Radiology Department, Assuit University Hospitals on 50 lesions, patient age ranging from 23 to 66 years old with mean age was $(46.9 \pm$ SD) years. MR imaging studies were performed using a $1.5 \mathrm{~T}$ Magnetom Vision scanner with dedicated bilateral phased-array breast coil (Siemens, Erlangen, Germany). MRI sequences were 1. Axial T1WI. 2. Axial T2WI. 3. Axial STIR. 4. Axial DWI and ADC. 5. DCE-MRI.

Results: The study included 50 lesions; 21 lesions were Luminal A, 15 lesions were Luminal B, 10 lesions were HER2+, and 4 lesions were TN. Histopathology 39 lesions were IDC-NOS, 1 lesion was IDC (medullary type), 7 lesions were mixed pathology IDC + DCIS, and 3 lesions were ILC. As regard the correlation between the histopathological type and grade, both were specific in differentiation between the molecular subtypes. According to the MRI findings it was found that T2 Intratumoral signal intensity, STIR, and the margin of the lesion were highly specific in differentiation, both L.N status and number were found that they were highly specific in differentiation ( $\mathrm{p}<0.001$ ), while size of the lesion, T1WI signal, ADC values were found that they were non-specific in differentiation between the molecular subtypes of breast cancer. Conclusion: Breast MRI may help in assessing different molecular subtypes of breast cancer.

Keywords: Molecular Subtype, Luminal A, Luminal B, HER2+, Triple-negative, MRI, ADC, DCE-MRI.

\section{Introduction}

Breast cancer is a collection of diseases defined by distinct pathological (e.g., ductal, lobular, mucinous) and molecular characteristics (e.g., estrogen receptor (ER) and progesterone receptor expression (PR),
HER2 amplification, and more recently transcriptome-based classifications like luminal and basal cancers (Jenkins et al., 2021). Initially, the WHO defined breast carcinoma by its morphological and immunohistochemical characteristics to Infiltrating ductal carcinoma (IDC), 
Infiltrating lobular carcinoma (ILC), Mucinous carcinoma, Medullary carcinoma, and Tubular carcinoma (Boisserie-Lacroix et al., 2013). Molecular subtyping is useful for the diagnosis and individualized treatment of breast cancer. However, the determination of subtypes by genetic analysis is invasive and expensive, requiring specialized equipment and technical expertise. Immunohistochemical surrogate biomarkers of estrogen receptor (ER), progesterone receptor (PR), and HER2 status are used to define molecular subtypes. Therefore, demand exists for alternative means of classifying breast cancers into distinct molecular subtypes. MRI has played an evolving role within the diagnosis and treatment of breast carcinoma (Ng et al., 2015). Regarding medical imaging, dynamic contrast-enhanced MRI (DCE-MRI) and diffusion-weighted imaging (DWI) are now both universally recognized and widely used modalities in multiparametric MRI (mpMRI) to diagnose and stage breast cancer, to assess post-chemotherapy response, and to differentiate between scar tissue and recurrent tumor (Fan et al., 2017). We aimed to assess the utility of MRI as an accurate method for detection of molecular subtypes of breast cancer by reaching our standard parameters for detection of these molecular subtypes.

\section{Patients and Methods}

Study design: A retrospective study.

Patients: A study of 2-years enrollment duration was conducted at Radiology Department, Assuit University Hospitals on 50 lesions, patient age ranging from 23 to 66 years old with mean age was $(46.9 \pm \mathrm{SD})$ years, 21 lesions (42.0\%) were Luminal A, 15 lesions (30.0\%)were Luminal B, 10 lesions (20.0\%) were HER-2, and 4 lesions $(8.0 \%)$ were Triple negative. As regard histopathology 39 lesions $(78.0 \%)$ were
IDC-NOS, 1 lesion (2.0\%) was IDC (medullary like), 7 lesions (14.0\%) were mixed pathology IDC + DCIS, and 3 lesions $(6.0 \%)$ were ILC.

Inclusion criteria include:

1) Patients diagnosed as breast cancer who had complete medical records, that include:

- Ultrasonography.

- $\quad$ Soft tissue mammography.

- $\quad$ MRI

- Biopsy with histopathology and immunohistochemistry.

2) Patients suffering from recurrence of breast cancer after treatment.

Exclusion criteria include:

3) Patients with history of breast implants.

\section{Methods}

- All patients were evaluated clinically if that they had pain, nipple discharge, skin retraction, and nipple retraction.

- MR imaging studies were performed employing a $1.5 \mathrm{~T}$ Magnetom Vision scanner with dedicated bilateral phased array breast coil (Siemens, Erlangen, Germany). Our MRI sequences were:

1. Axial T1WI. 2. Axial T2WI. 3. Axial STIR. 4. Axial DWI and ADC. 5. DCEMRI.

\section{MRI Acquisition protocol:}

a. Conventional MRI protocols; included T1-weighted sequences (TR 6.1, TE 2.6, TI 600, NEX 1, flip angle 15 and $4 \mathrm{~mm}$ slice thickness). T2- weighted axial sequences (TR 4500, TE 70, NEX 1,flip angle 120 and $4 \mathrm{~mm}$ slice thickness), and STIR images (TR 8.75, TE 4.33, NEX 1, flip angle 15 and $0.9 \mathrm{~mm}$ slice thickness).

b. Functional MRI; DWI echo-planar images (TR/TE 8500/70, FOV of $330 \mathrm{~mm}$, matrix $192 \times 192$, NEX: 1 , sectional thickness $4.5 \mathrm{~mm}$ with a 1 
mm intersection gap) were obtained within the axial plane before contrast administration.DWI were obtained by diffusion gradients between 0 and $2000 \mathrm{sec} / \mathrm{mm} 2 \mathrm{~b}$-values.

An ADC map was automatically constructed in an exceedingly commercially available workstation. Mean ADC values of all lesions were automatically measured by using these maps in line with the formula $\mathrm{ADC}=(\operatorname{lnS} 0-\ln S) / \mathrm{b}($ signal intensity values are measured as $\mathrm{S} 0$ at $\mathrm{b}=0$ $\mathrm{sec} / \mathrm{mm} 2$ and $\mathrm{S}$ at $\mathrm{b}=2000$ $\mathrm{sec} / \mathrm{mm} 2$ ).

The standard dynamic protocol started with an unenhanced coronal three-dimensional fast field echo (thrive) sense T1-weighted sequence. A bolus $(14 \mathrm{ml})$ of gadolinium containing contrast $(0.1 \mathrm{mmol} / \mathrm{kg})$ was administered intravenously at 3 $\mathrm{ml} / \mathrm{sec}$ by employing a power injector followed by a bolus of $30 \mathrm{ml}$ of isotonic solution. Subsequently, dynamic imaging was performed in five consecutive series at 90-second intervals. The voxel size was $1.21 \mathrm{X}$ $1.21 \mathrm{X} 1.69 \mu 1$. The subsequent scanning parameters were used: acquisition time of 90 seconds; ratio of repetition time to echo time, 8.1:4.0; flip angle, 20 degrees; field of view, $310 \mathrm{~mm}$.

- MRI Findings: The lesion type, in line with BI-RADS was classified into mass or non-mass-likeenhancement. If the lesion was mass it had been evaluated in keeping with its size, shape, margin, T1, T2
Intratumoral signal intensity, skin thickening, skin invasion, nipple invasion, L.N status and if positive; L.N number., while within the nonmass-like-enhancement were studied the distribution modifiers and internal enhancement pattern, skin thickening, skin invasion, nipple invasion, L.N status and if positive; L.N number.

Statistical Analysis: Data were collected and entered to the pc using SPSS (Statistical Package for Social Science) program for statistical analysis. Data were entered as numerical or categorical, as appropriate. To match the MRI findings, mass or nonmasslike-enhancement, between the subtypes of breast carcinoma, we used the chi-square test $\left(\mathrm{v}^{2}\right)$ with $\mathrm{p}<0.05$ considered to point a big difference.

\section{Results}

The study included 50 lesions; 21 lesions(42.0\%) were Luminal A, 15 lesions (30.0\%)were Luminal B, 10 lesions $(20.0 \%)$ were HER2+, and 4 lesions (8.0\%)were Triple negative (Table 1).

Clinical evaluation of the patients in line with their age, pain, nipple discharge, skin retraction, and nipple retraction (Table 2), it absolutely was found that there was nonsignificant difference between groups of molecular subtypes as regard age of patients. However there was significant difference between groups of molecular subtypes as regard pain, nipple discharge and skin retraction. 
Table 1. Classification and Distribution of Breast Cancer Subtype by ER, PR, and HER2 status

\begin{tabular}{|c|c|c|c|c|}
\hline Subtype & ER and/or PR & HER2+ & n & \% \\
\hline Luminal A & Positive & Negative & 21 & 42 \\
\hline Luminal B & Positive & Positive & 15 & 30 \\
\hline HER2 & Negative & Positive & 10 & 20 \\
\hline Triple negative & Negative & Negative & 4 & 8 \\
\hline Total & & & 50 & 100 \\
\hline
\end{tabular}

Table 2. Clinical features in different molecular subtypes

\begin{tabular}{|c|c|c|c|c|c|c|c|}
\hline Variables & & $\begin{array}{c}\text { Luminal } \\
\text { A }\end{array}$ & Luminal B & HER2+ & TN & $\begin{array}{c}\text { Chi } \\
\text { square } \\
\text { or } \\
\text { ANOV } \\
\text { A }^{*}\end{array}$ & P value \\
\hline Age & $\begin{array}{c}\text { Mean } \\
\pm \text { SD }\end{array}$ & $\begin{array}{c}46.76 \pm 5 . \\
84\end{array}$ & $\begin{array}{c}47.00 \pm 14.6 \\
6\end{array}$ & $52.00 \pm 7.37$ & $\begin{array}{c}44.00 \pm 14 . \\
00\end{array}$ & $0.853^{*}$ & $\begin{array}{c}0.472 \\
(\mathrm{NS})\end{array}$ \\
\hline \multirow[t]{2}{*}{ Pain } & Yes & $\begin{array}{c}5 \\
(23.8 \%)\end{array}$ & $10(66.7 \%)$ & $5(50 \%)$ & $1(25 \%)$ & \multirow[t]{2}{*}{7.336} & \multirow[t]{2}{*}{$0.057(\mathrm{~S})$} \\
\hline & No & $\begin{array}{c}16 \\
(76.2 \%)\end{array}$ & $5(33.3 \%)$ & $5(50 \%)$ & $3(75 \%)$ & & \\
\hline \multirow[t]{2}{*}{$\begin{array}{c}\text { Nipple } \\
\text { discharge }\end{array}$} & Yes & $\begin{array}{c}6 \\
(28.6 \%)\end{array}$ & $0(0 \%)$ & $5(50 \%)$ & $1(25 \%)$ & \multirow[t]{2}{*}{8.686} & \multirow[t]{2}{*}{$0.009(\mathrm{~S})$} \\
\hline & No & $\begin{array}{c}15 \\
(71.4 \%)\end{array}$ & $15(100 \%)$ & $5(50 \%)$ & $3(75 \%)$ & & \\
\hline \multirow[t]{2}{*}{$\begin{array}{c}\text { Skin } \\
\text { retraction }\end{array}$} & Yes & $\begin{array}{c}3 \\
(14.3 \%)\end{array}$ & $5(33.3 \%)$ & $10(100 \%)$ & $0(0 \%)$ & \multirow[t]{2}{*}{24.372} & \multirow[t]{2}{*}{$\begin{array}{c}<0.001 \\
(\mathrm{HS})\end{array}$} \\
\hline & No & $\begin{array}{c}18 \\
(85.7 \%)\end{array}$ & $10(66.7 \%)$ & $0(0 \%)$ & $4(100 \%)$ & & \\
\hline \multirow[t]{2}{*}{$\begin{array}{c}\text { Nipple } \\
\text { retraction }\end{array}$} & Yes & $\begin{array}{c}12 \\
(57.1 \%)\end{array}$ & $5(33.3 \%)$ & $5(50 \%)$ & $0(0 \%)$ & \multirow[t]{2}{*}{5.454} & \multirow[t]{2}{*}{$\begin{array}{c}0.073 \\
\text { (NS) }\end{array}$} \\
\hline & No & $\begin{array}{c}9 \\
(42.9 \%) \\
\end{array}$ & $10(66.7 \%)$ & $5(50 \%)$ & $4(100 \%)$ & & \\
\hline
\end{tabular}


Table 3. Histopathological typeof lesions with LA, LB, HER2+, and TNBC breast cancer subtypes

\begin{tabular}{|c|c|c|c|c|c|c|}
\hline \multirow{2}{*}{ Histolpathology } & \multicolumn{5}{|c|}{ Immuno type } & \multirow{2}{*}{ Total } \\
\cline { 2 - 6 } & HER2+ & LA & LB & TN & 39 \\
IDC-NOS & Count & 10 & 16 & 10 & 3 & $78.0 \%$ \\
\hline \% within Immuno type & $100.0 \%$ & $76.2 \%$ & $66.7 \%$ & $75.0 \%$ & 7 \\
\hline IDC (medullary) Count & 0 & 0 & 0 & 1 & 1 \\
& $\%$ within Immuno type & $0.0 \%$ & $0.0 \%$ & $0.0 \%$ & $25.0 \%$ & $2.0 \%$ \\
\hline IDC + DCIS & Count & 0 & 2 & 5 & 0 & 7 \\
& \% within Immuno type & $0.0 \%$ & $9.5 \%$ & $33.3 \%$ & $0.0 \%$ & $14.0 \%$ \\
\hline ILC & Count & 0 & 3 & 0 & 0 & 3 \\
& $\%$ within Immuno type & $0.0 \%$ & $14.3 \%$ & $0.0 \%$ & $0.0 \%$ & $6.0 \%$ \\
\hline Total & Count & 10 & 21 & 15 & 4 & 50 \\
& $\%$ within Immuno type & $100.0 \%$ & $100.0 \%$ & $100.0 \%$ & $100.0 \%$ & $100.0 \%$ \\
\hline
\end{tabular}

Chi square $=22.789, P$ value $=0.03(S)$

As regard histopathological type 39 lesions $(78.0 \%)$ were IDC-NOS, 1 lesion $(2.0 \%)$ was IDC (medullarylike), 7 lesions (14.0\%) Histopathological type IDC-NOS was found in 39 lesions $(78.0 \%) 16$ lesion were LA, 10 lesions were LB, 10 lesions were HER2+, and three lesions were TN. IDC (medullary type) was found in 1 lesion (2.0\%) which was TN subtype. Mixed pathology IDC+DCIS was found in 7 lesions (14.0\%), during which 2 lesions were LA and 5 lesions were LB. ILC was found in three lesions $(6.0 \%)$, these lesions were LA subtype (Fig.1).So, it found that from total 21 lesions with LA subtype; 16 lesions (76.2\%) were IDC-NOS, 2 lesions $(9.5 \%)$ were mixed pathology (IDC+DCIS), and three lesions (14.3\%) were ILC however no lesions $(0.0 \%)$ were IDC (medullary type). From total 15 lesions with LB subtype; 10 lesions (66.7\%) were IDC-NOS, 5 lesions (33.3\%) were mixed pathology (IDC+DCIS), however no lesions $(0.0 \%)$ were IDC (medullary type) or ILC. From total 10 lesions with HER2+ subtype; all 10 lesions (100\%) were IDC-NOS, and from were mixed pathology IDC + DCIS, and three lesions $(6.0 \%)$ were ILC (Table 3).

total 4 lesions with TN subtype; 3 lesions (75.0\%) were IDC-NOS and 1 lesion $(25.0 \%)$ was IDC (medullary type).

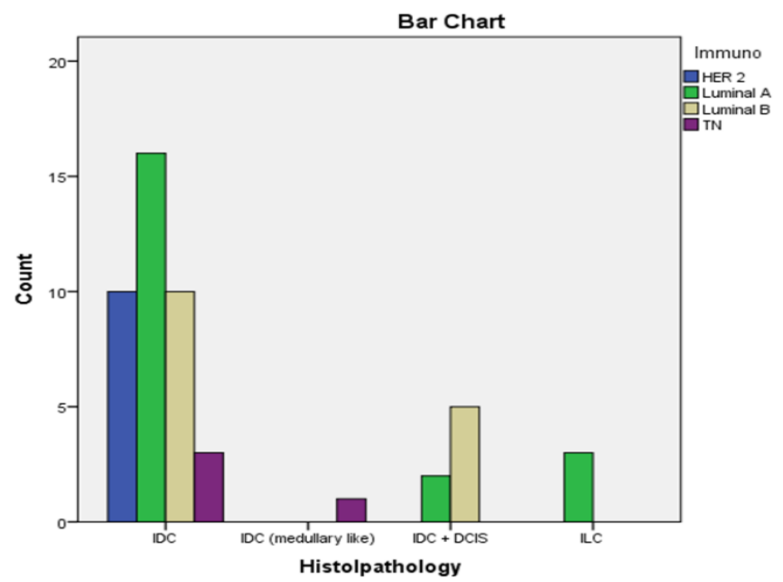

Fig. 1. There was significant difference between groups of molecular subtypes as regard histopathological type 
Table 4. Histopathological grade of lesions with LA, LB, HER2+, and TNBC breast cancer subtypes

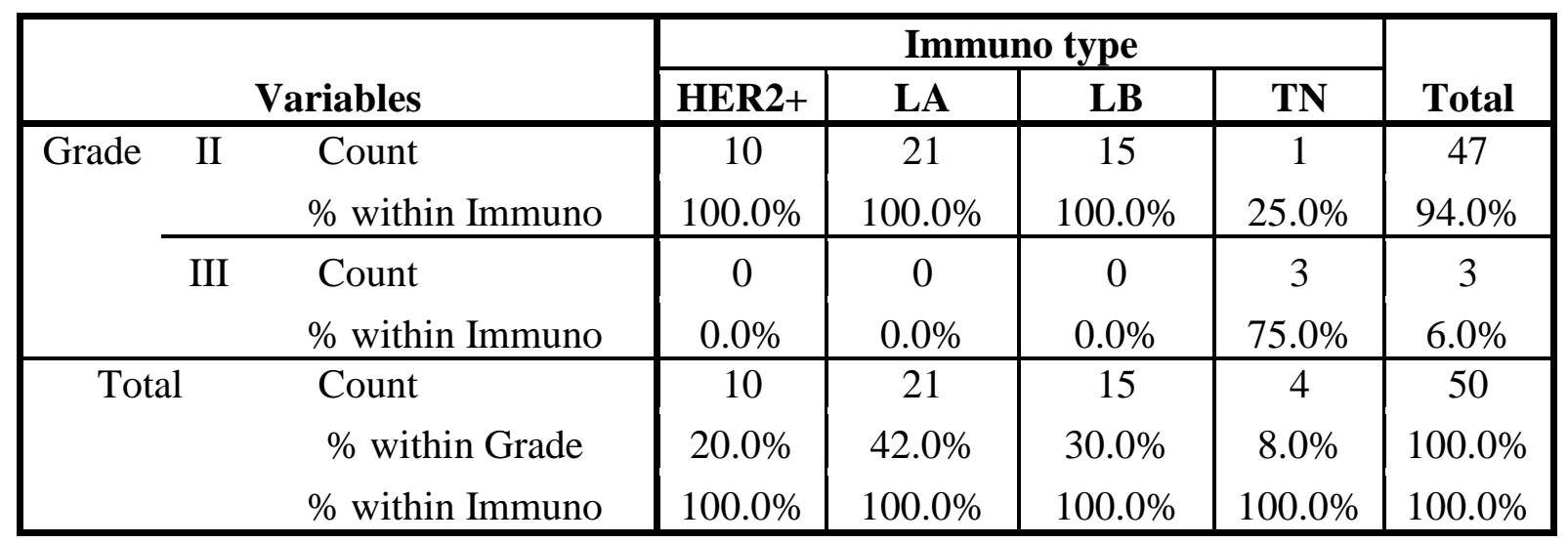

Chi square $=36.702$, $\mathrm{P}$ value $<0.001$ (HS)

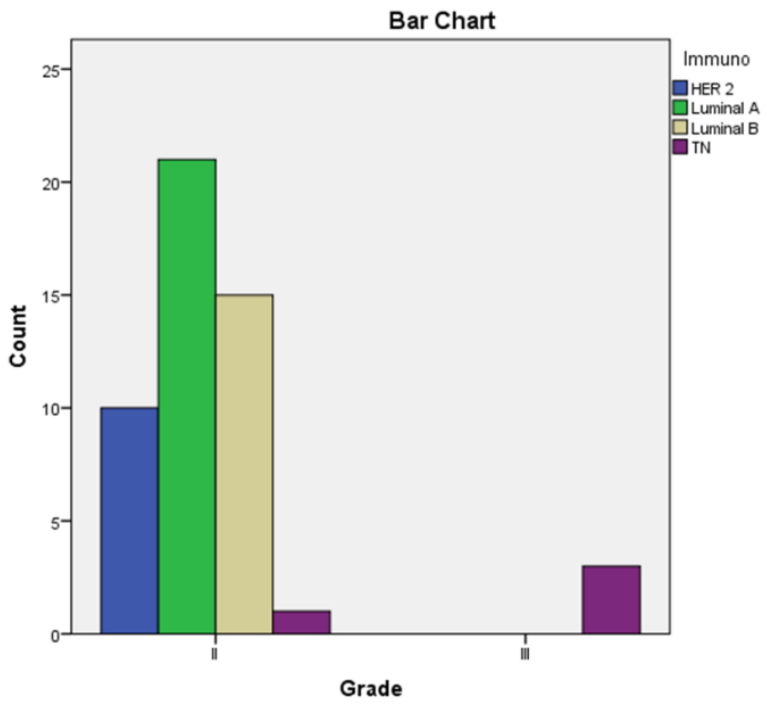

Fig. 2. There was significant difference between groups of molecular subtypes as regard histopathological grading.

Histologically grade II was found in $(94.0 \%)$, and grade III in $(6.0 \%)$ cases. Regarding the results comparing the pathological variable among the four tumor subtypes (Table 3), tumor histological grade was significantly different among them. The proportion of histological grade II in LA (42.0\%) was quite high as compared to TN (8.0\%), while histological grade III in TN was high compared to other types (Fig.2).

As regard MRI findings, all tumors were detected as an abnormal enhancement. The main bulk of the lesionsshowed mass-like enhancement $(96.0 \%)$ with different subtypes while only (4.0\%) showed nonmass enhancement during which their molecular subtype were LA, there was no significant difference between different subtypes as regard tumor size and T1WI,however there was significant difference between molecular subtypes as regard T2 Intratumoral signal intensity (Table 5) and STIR (Table 6), in TN breast carcinoma the lesion showed a high T2 signal intensity (Fig.3). 
Table 5. T2 Intratumoral signal intensity in different molecular subtypes

\begin{tabular}{|c|c|c|c|c|c|c|}
\hline \multirow{2}{*}{\multicolumn{3}{|c|}{ Variables }} & \multicolumn{4}{|c|}{ Immuno } \\
\hline & & & \multirow{2}{*}{$\frac{\text { HER2+ }}{7}$} & \multirow{2}{*}{\begin{tabular}{|c|}
$\mathbf{L A}$ \\
16
\end{tabular}} & \multirow{2}{*}{$\begin{array}{c}\text { LB } \\
6\end{array}$} & \multirow{2}{*}{$\begin{array}{c}\text { TN } \\
0\end{array}$} \\
\hline \multirow{5}{*}{$\begin{array}{c}\text { T2 } \\
\text { Intratum } \\
\text { oral } \\
\text { signal } \\
\text { intensity }\end{array}$} & \multirow[t]{2}{*}{ Hypointense } & Count & & & & \\
\hline & & $\begin{array}{l}\% \text { within } \\
\text { Immuno }\end{array}$ & $70.0 \%$ & $84.2 \%$ & $54.6 \%$ & $0.0 \%$ \\
\hline & \multicolumn{2}{|c|}{$\begin{array}{ll}\text { Isointense } & \text { Count } \\
& \% \text { within Immuno }\end{array}$} & $\begin{array}{c}0 \\
0.0 \%\end{array}$ & $\begin{array}{c}3 \\
15.8 \%\end{array}$ & $\begin{array}{c}5 \\
45.5 \%\end{array}$ & $\begin{array}{c}1 \\
25.0 \%\end{array}$ \\
\hline & \multirow{2}{*}{$\begin{array}{l}\text { Hyperintense/ Very } \\
\text { hyperintense }\end{array}$} & Count & 3 & 0 & 0 & 3 \\
\hline & & $\begin{array}{l}\% \text { within } \\
\text { Immuno }\end{array}$ & $30.0 \%$ & $0.0 \%$ & $0.0 \%$ & $75.0 \%$ \\
\hline \multirow{2}{*}{\multicolumn{2}{|c|}{ Total }} & Count & 10 & 19 & 11 & 4 \\
\hline & & $\begin{array}{l}\text { \% within } \\
\text { Immuno }\end{array}$ & $100.0 \%$ & $100.0 \%$ & $100.0 \%$ & $100.0 \%$ \\
\hline
\end{tabular}

Chi square $=103.226, P$ value $<0.001(\mathrm{HS})$

Table 6. STIR signal in different molecular subtypes

\begin{tabular}{|c|c|c|c|c|c|c|c|}
\hline \multirow{2}{*}{\multicolumn{3}{|c|}{ Variables }} & \multicolumn{4}{|c|}{ Immuno } & \multirow[b]{2}{*}{ Total } \\
\hline & & & HER2+ & LA & LB & TN & \\
\hline \multirow{10}{*}{$\begin{array}{l}\text { STI } \\
\mathbf{R}\end{array}$} & \multirow{2}{*}{$\begin{array}{c}\text { Heterogenous } \\
\text { predominantly Hyperintense }\end{array}$} & Count & 8 & 18 & 6 & 2 & 34 \\
\hline & & $\begin{array}{l}\% \text { within } \\
\text { Immuno }\end{array}$ & $80.0 \%$ & $94.7 \%$ & $54.5 \%$ & $50.0 \%$ & $77.3 \%$ \\
\hline & \multirow{2}{*}{$\begin{array}{c}\text { Heterogenous } \\
\text { predominantly hyperintense } \\
\text { with signal void foci }\end{array}$} & Count & 2 & 0 & 0 & 1 & 3 \\
\hline & & $\begin{array}{l}\% \text { within } \\
\text { Immuno }\end{array}$ & $20.0 \%$ & $0.0 \%$ & $0.0 \%$ & $25.0 \%$ & $6.8 \%$ \\
\hline & \multirow{2}{*}{$\begin{array}{l}\text { Hyperintense cystic and } \\
\text { heterogenous solid } \\
\text { component }\end{array}$} & Count & 0 & 0 & 0 & 1 & 1 \\
\hline & & $\begin{array}{l}\% \text { within } \\
\text { Immuno }\end{array}$ & $0.0 \%$ & $0.0 \%$ & $0.0 \%$ & $25.0 \%$ & $2.3 \%$ \\
\hline & \multirow{2}{*}{$\begin{array}{c}\text { Hyperintense with central } \\
\text { signal } \\
\text { void foci }\end{array}$} & Count & 0 & 1 & 0 & 0 & 1 \\
\hline & & $\begin{array}{l}\text { \% within } \\
\text { Immuno }\end{array}$ & $0.0 \%$ & $5.3 \%$ & $0.0 \%$ & $0.0 \%$ & $2.3 \%$ \\
\hline & \multirow{2}{*}{$\begin{array}{l}\text { Isointense with foci of } \\
\text { hyperintense signal }\end{array}$} & Count & 0 & 0 & 5 & 0 & 5 \\
\hline & & \begin{tabular}{|l|}
$\%$ within \\
Immuno
\end{tabular} & $0.0 \%$ & $0.0 \%$ & $45.5 \%$ & $0.0 \%$ & $11.4 \%$ \\
\hline \multirow{2}{*}{\multicolumn{2}{|c|}{ Total }} & Count & 10 & 19 & 11 & 4 & 44 \\
\hline & & $\begin{array}{l}\text { \% within } \\
\text { Immuno }\end{array}$ & $100.0 \%$ & $100.0 \%$ & $100.0 \%$ & $100.0 \%$ & $100.0 \%$ \\
\hline \multicolumn{8}{|c|}{ Chi square $=34.729$, P value $=0.004(S)$} \\
\hline
\end{tabular}


(a)

(b)

(c)
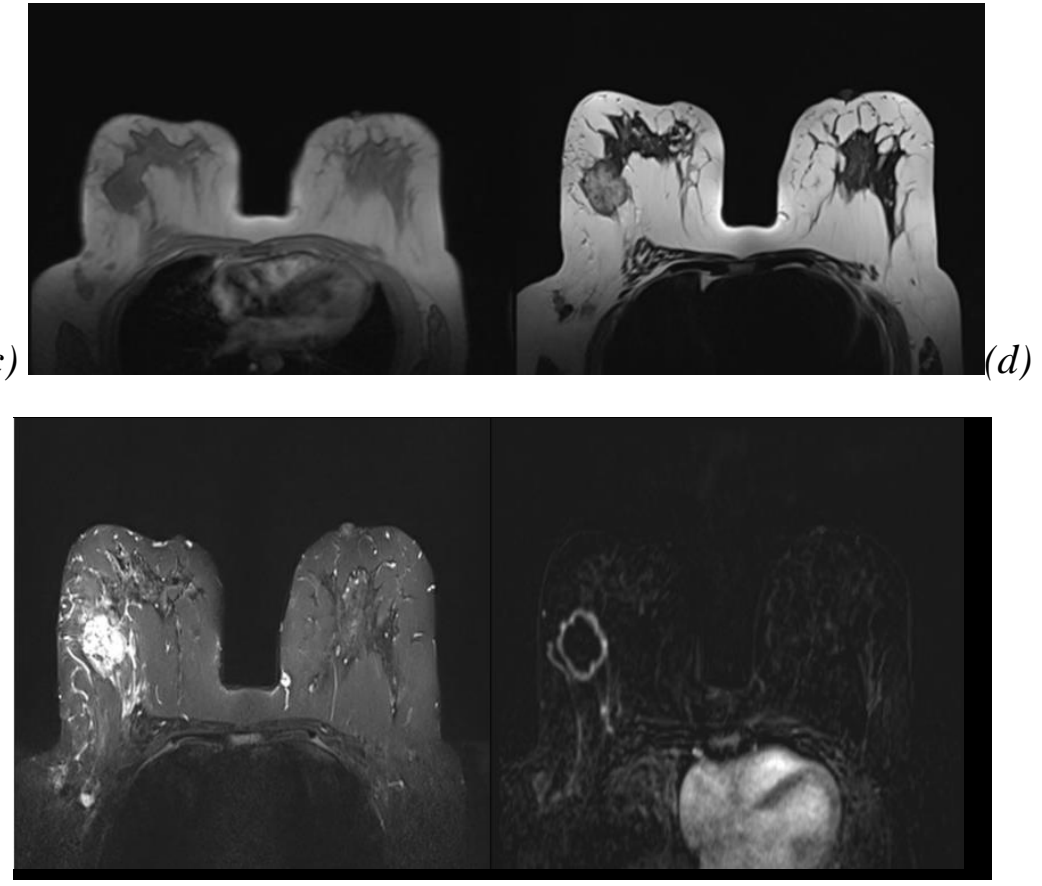

(e)

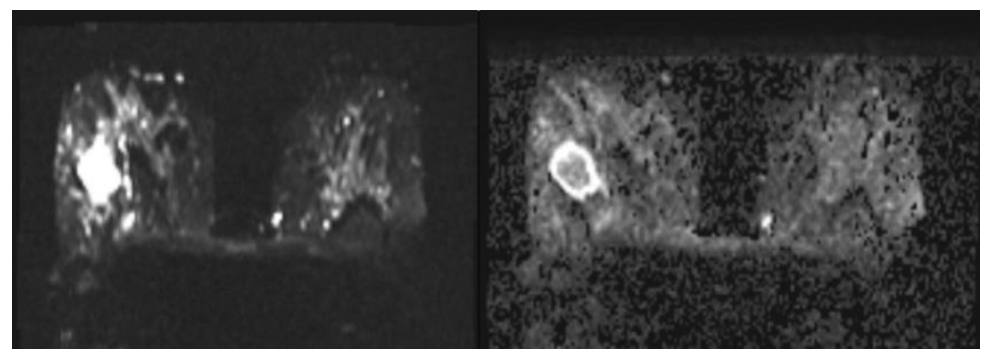

(f)

Fig.5.51 years old female patient, presented by right breast lump with TNBC subtype and grade III IDC-NOS, T1 (a) hypointense, T2 (b) image shows a hyperintense rounded lesion with smooth margin, STIR (c) shows hyperintense signal, subtraction image (d) shows ring enhancement, DWI (e) of high signal (restricted) with ADC signal (f) of low signal in the center and high signal peripherally. 
Table 7. Margin of the lesion in different molecular subtypes:

\begin{tabular}{|c|c|c|c|c|c|c|}
\hline \multirow{2}{*}{\multicolumn{2}{|c|}{ Variables }} & \multicolumn{4}{|c|}{ Immuno } & \multirow[b]{2}{*}{ Total } \\
\hline & & \multirow{2}{*}{$\frac{\text { HER 2+ }}{0}$} & \multirow{2}{*}{$\frac{\text { LA }}{0}$} & \multirow{2}{*}{$\frac{\mathbf{L B}}{0}$} & \multirow{2}{*}{$\frac{\mathbf{T N}}{1}$} & \\
\hline Margin & Count & & & & & 1 \\
\hline Circumscribed & $\%$ within Immuno & $0.0 \%$ & $0.0 \%$ & $0.0 \%$ & $25.0 \%$ & $2.3 \%$ \\
\hline \multirow[t]{2}{*}{ Irregular } & Count & 5 & 4 & 9 & 0 & 18 \\
\hline & $\%$ within Immuno & $50.0 \%$ & $21.1 \%$ & $81.8 \%$ & $0.0 \%$ & $40.9 \%$ \\
\hline \multirow[t]{2}{*}{ Lobulated } & Count & 4 & 3 & 0 & 0 & 7 \\
\hline & $\%$ within Immuno & $40.0 \%$ & $15.8 \%$ & $0.0 \%$ & $0.0 \%$ & $15.9 \%$ \\
\hline \multirow[t]{2}{*}{ Spiculated } & Count & 1 & 12 & 2 & 3 & 18 \\
\hline & $\%$ within Immuno & $10.0 \%$ & $63.2 \%$ & $18.2 \%$ & $75.0 \%$ & $40.9 \%$ \\
\hline \multirow[t]{2}{*}{ Total } & Count & 10 & 19 & 11 & 4 & 44 \\
\hline & $\%$ within Immuno & $100.0 \%$ & $100.0 \%$ & $100.0 \%$ & $100.0 \%$ & $100.0 \%$ \\
\hline
\end{tabular}

Chi square $=31.364, P$ value $<0.001(\mathrm{HS})$

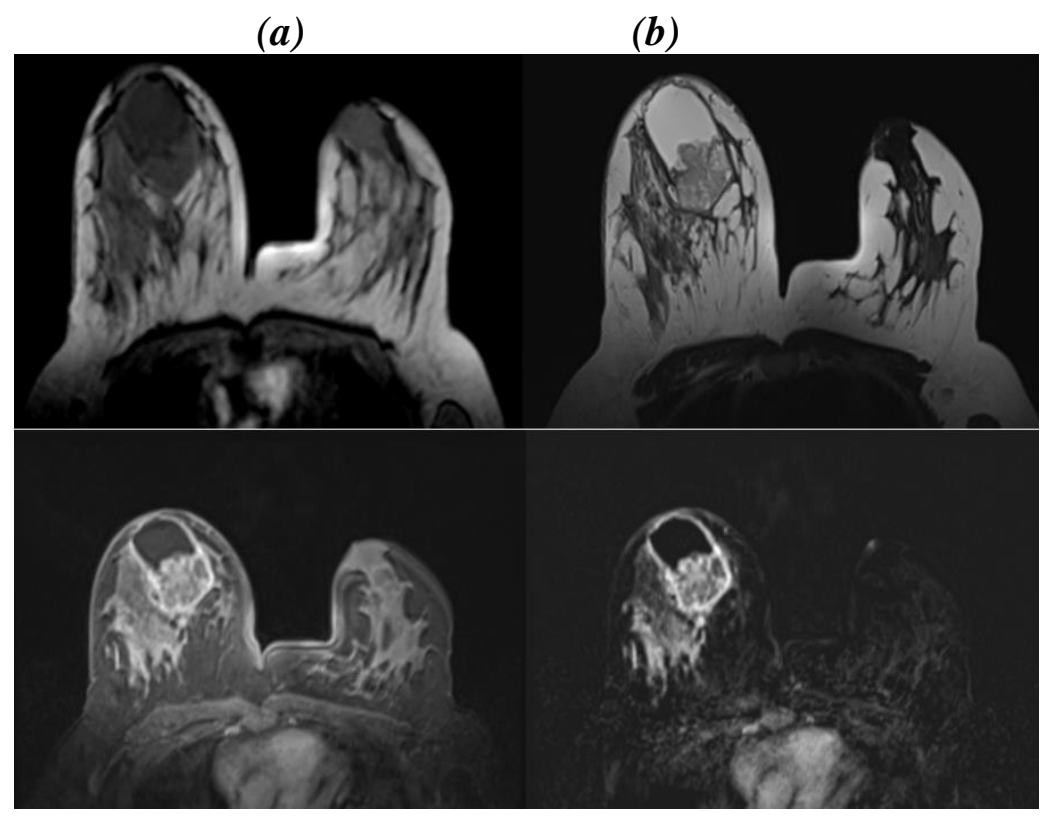

(c)

(d)

Fig.4. 23 years old female patient, presented by painful lump at the right breast of 3 month duration with TNBC subtype and grade II IDC (medullary type), T1 (a) hypointense, T2 (b) image shows a hyperintense oval lesion with smooth circumscribed margin in the right breast, Dynamic postcontrast enhancement (c) and subtraction image (d) shows ring enhancement of the mass with heterogeneous enhancement of the cystic component.

The margin of the lesion was found that it absolutely was highly specific in differentiation ( $\mathrm{p}$ <0.001) (Table 7), circumscribed lesions seen only in $\mathrm{TN}$ breast 
carcinoma (fig.4), while spiculated lesions seen mostly in LA subtype (fig. 5), there was significant difference between molecular subtypes of breast carcinoma as regard both L.N status (fig.6) and L.Ns number (fig.7). (a)

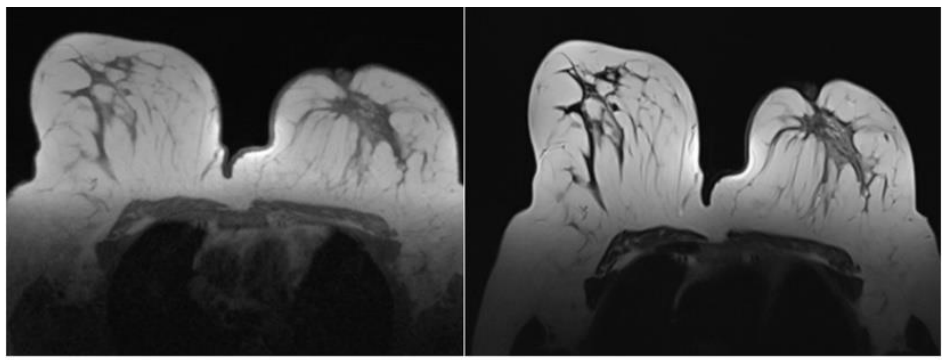

(c)

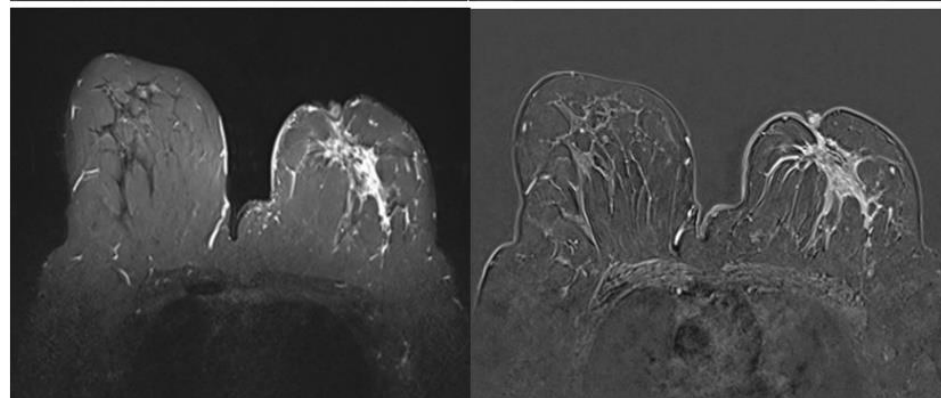

(d)

Fig.5. 42 years old female patient, presented by painless lump at the left breast with LA subtype and grade II ILC, T1 (a) isointense, T2 (b) image shows a speculated mixed iso and hypointense lesion, STIR (c) heterogenous intensity and subtraction (d) mild heterogeneous enhancement.

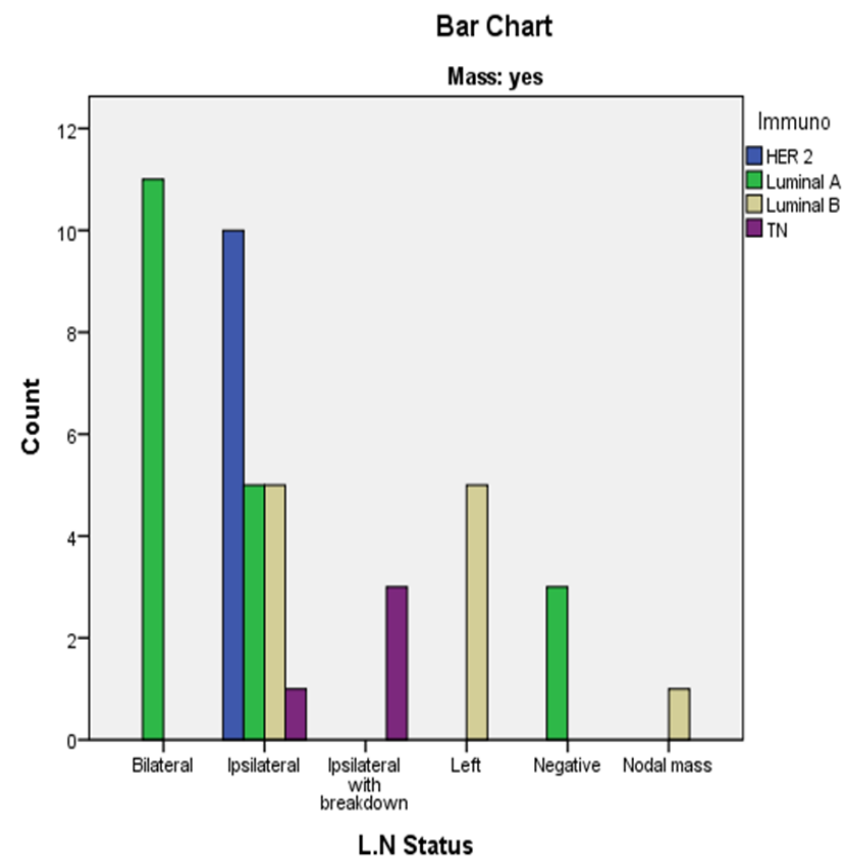

Fig.6. L.N status in different molecular subtypes. 


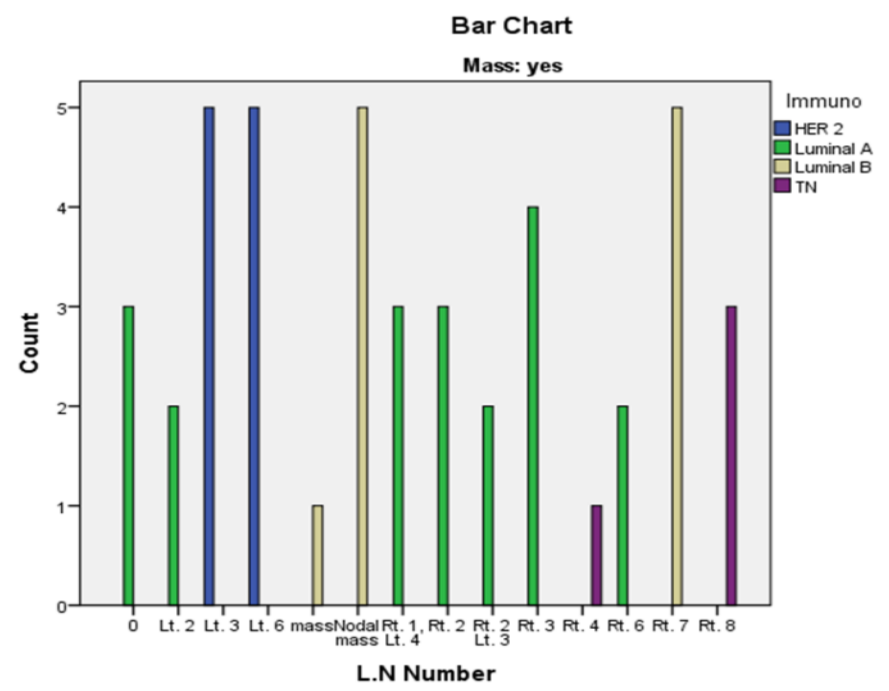

Fig. 7. L.N number in different molecular subtypes.

Table 8. Different ADC Values in different molecular subtypes:

\begin{tabular}{|c|c|c|c|c|c|c|}
\hline \multirow{2}{*}{\multicolumn{2}{|c|}{ Variables }} & \multicolumn{4}{|c|}{ Immunotype } & \multirow{2}{*}{ Total } \\
\hline & & \multirow{2}{*}{$\begin{array}{c}\text { HER2 } \\
+ \\
0 \\
0.0 \% \\
\end{array}$} & \multirow{2}{*}{$\begin{array}{c}\text { LA } \\
1 \\
4.8 \% \\
\end{array}$} & \multirow{2}{*}{$\begin{array}{c}\mathbf{L B} \\
0 \\
0.0 \% \\
\end{array}$} & \multirow{2}{*}{$\begin{array}{c}\mathbf{T N} \\
0 \\
0.0 \% \\
\end{array}$} & \\
\hline $\begin{array}{c}\text { ADC Value } 0.4 \times 10^{-3} \\
\mathrm{~mm}^{2} / \mathrm{s}\end{array}$ & $\begin{array}{c}\text { Count } \\
\text { \% within Immuno }\end{array}$ & & & & & $\begin{array}{c}1 \\
2.0 \%\end{array}$ \\
\hline $0.5 \times 10^{-3} \mathrm{~mm}^{2} / \mathrm{s}$ & $\begin{array}{c}\text { Count } \\
\% \text { within Immuno } \\
\end{array}$ & $\begin{array}{c}2 \\
20.0 \% \\
\end{array}$ & $\begin{array}{c}2 \\
9.5 \% \\
\end{array}$ & $\begin{array}{c}2 \\
13.3 \% \\
\end{array}$ & $\begin{array}{c}0 \\
0.0 \% \\
\end{array}$ & $\begin{array}{c}6 \\
12.0 \% \\
\end{array}$ \\
\hline $0.6 \times 10^{-3} \mathrm{~mm}^{2} / \mathrm{s}$ & $\begin{array}{c}\text { Count } \\
\text { \% within Immuno }\end{array}$ & $\begin{array}{c}1 \\
10.0 \%\end{array}$ & $\begin{array}{c}3 \\
14.3 \% \\
\end{array}$ & $\begin{array}{c}2 \\
13.3 \% \\
\end{array}$ & $\begin{array}{c}1 \\
25.0 \% \\
\end{array}$ & $\begin{array}{c}6 \\
12.0 \% \\
\end{array}$ \\
\hline $0.7 \times 10^{-3} \mathrm{~mm}^{2} / \mathrm{s}$ & $\begin{array}{c}\text { Count } \\
\text { \% within Immuno }\end{array}$ & $\begin{array}{c}6 \\
60.0 \% \\
\end{array}$ & $\begin{array}{r}7 \\
33.3 \% \\
\end{array}$ & $\begin{array}{c}3 \\
20 \% \\
\end{array}$ & $\begin{array}{c}3 \\
75.0 \% \\
\end{array}$ & $\begin{array}{c}16 \\
32.0 \% \\
\end{array}$ \\
\hline $0.8 \times 10^{-3} \mathrm{~mm}^{2} / \mathrm{s}$ & $\begin{array}{c}\text { Count } \\
\text { \% within Immuno }\end{array}$ & $\begin{array}{c}1 \\
10.0 \% \\
\end{array}$ & $\begin{array}{c}4 \\
19.0 \% \\
\end{array}$ & $\begin{array}{c}4 \\
26.7 \% \\
\end{array}$ & $\begin{array}{c}0 \\
0.0 \% \\
\end{array}$ & $\begin{array}{c}9 \\
18.0 \% \\
\end{array}$ \\
\hline $0.9 \times 10^{-3} \mathrm{~mm}^{2} / \mathrm{s}$ & $\begin{array}{c}\text { Count } \\
\text { \% within Immuno } \\
\end{array}$ & $\begin{array}{c}0 \\
0.0 \% \\
\end{array}$ & $\begin{array}{c}4 \\
19.0 \%\end{array}$ & $\begin{array}{c}4 \\
26.7 \%\end{array}$ & $\begin{array}{c}0 \\
0.0 \%\end{array}$ & $\begin{array}{c}8 \\
16.0 \%\end{array}$ \\
\hline Total & $\begin{array}{c}\text { Count } \\
\% \text { within Immuno }\end{array}$ & $\begin{array}{c}10 \\
100.0 \\
\%\end{array}$ & $\begin{array}{c}21 \\
100.0 \%\end{array}$ & $\begin{array}{c}15 \\
100.0 \%\end{array}$ & $\begin{array}{c}4 \\
100.0 \%\end{array}$ & $\begin{array}{c}50 \\
100.0 \%\end{array}$ \\
\hline
\end{tabular}

Chi square $=38.728, P$ value $=0.089(\mathrm{NS})$ 
There was no significant difference in differentiation between molecular subtypes as regard DWI and ADC values (Table 8).

\section{Discussion}

Breast cancer can have a variable biological behavior and aggressiveness, Molecular subtypes of breast carcinoma incorporates a different pattern of clinical presentation and imaging findings, Regarding medical imaging, dynamic contrast-enhanced MRI (DCE-MRI) and diffusion-weighted imaging (DWI) are now both universally recognized and widely used modalities in multiparametric MRI (mp-MRI) to diagnose and stage breast carcinoma, to assess postchemotherapy response, and to differentiate between scarred tissue and recurrent tumor (Allarakha et al., 2019). Our study may help in assessing different MRI features that helpful in differentiating between molecular subtypes of breast carcinoma, which might be helpful in improvement of the selection of presurgical neoadjuvant therapy.

In our study, we couldn't find significant age differences among different subtypes as compared to previous study conducted by Osman NM et al where it absolutely was found that TNBC was more common at a younger age $(43.1 \pm 8.2)$ as compared to ER $(45 \pm 6.1)$ and HER2+ (47.4 \pm 6.6) (Osman et al., 2014).

Our study showed that histologically high grade tumors were more common in TN subtype as compared to other subtypes which was not in keeping with previous studies conducted by Issar $\mathrm{P}$ et al where it absolutely was found that histologically high grade tumors were more common in LB, HER2+ and TNBC subtypes as compared to LA (Issaret al., 2020).

In our study we found that TN lesions presented by smooth circumscribed margin which is analogous to the study conducted by Schrading and Kuhl (Schrading et al., 2008), who reported that familial breast carcinoma tends to exhibit smooth mass margins. Accordingly, specific subtypes of high-grade tumors, like triplenegative and familial breast cancers are likely to manifest with benign morphologic features (Schrading et al., 2008; Navarro et al., 2017).

We found that medullary carcinoma was significantly related to triple-negative subtype, which is in keeping with previous studies (Navarro et al., 2017; Uematsu et al., 2009).

Luminal A tumors were more common in our study (42.0\%), presenting as a mass lesion with an irregular shape, speculated margin, and heterogeneous enhancement. These observations were just like the study conducted by Youk JH et al (Youk et al., 2012).Overall, Luminal A carcinoma is related to the foremost favorable prognosis. Grimm et al. and Agarwal $\mathrm{G}$ et al. reported that Luminal B and HER2 positive tumors were related to axillary adenopathy as compared to LA and $\mathrm{TN}$ which indicate a more invasive behavior and greater metastatic potential (Grimm et al., 2014).

TN carcinoma showed a high T2 signal intensity $(75.0 \%)$ in our study as compared to $(72.73 \%)$ within the study conducted by Issar $\mathrm{P}$ et al (Issar et al., 2020), also Azzam et al concluded that intratumoral bright signal intensity on T2weighted images; seen in TN carcinomawhere about $(62.8 \%)$ of $\mathrm{TN}$ lesions elicited high T2 signal intensity, and also it's accompanied with more aggressive tumors(Azzam et al., 2019).The hyperintense signal corresponded to intratumoral necrosis, which could be a prognostic factor in invasive carcinoma. It's reported that the presence of moderate to 
marked central tumor necrosis decreases relapse-free survival and increases mortality in both patients with node-negative and node-positive disease. Centrally necrotizing breast cancers were characterized by early systemic metastasis and an accelerated clinical course (Issaret al., 2020; Sung et al., 2013).

In this study there was no significant difference in differentiation between molecular subtypes as regard ADC values, this was the same as the study conducted by Surov et al, which concluded that there was no significant differences of ADC values were observed between luminal A, HER 2+ and triple negative tumors, while Luminal B carcinomas had statistically significant lower ADC values compared with luminal $A$ $(p=0.003)$ and HER $2+(p=0.007)$ lesions (Surov et al., 2019), that's wasn't the same as the previous studies conducted by Youk et al, who found that TN carcinoma showed higher ADC values that's explained by the high or very high intratumoral signal intensity on T2-weighted MRI showed a considerable correlation with ADC value $(\mathrm{P}=0.001)$ and was observed more frequently in TNBC than in other tumor subtypes $(\mathrm{P}<0.0001)$ because of tumor necrosis(Youk et al., 2012).

\section{Conclusion}

MR imaging can help in differentiating between molecular subtypes of breast carcinoma, In Luminal Atumors which might be presented as a mass with a speculated margin, while Luminal B showed irregular margin. TN breast carcinoma presents several MRI predictors on DCEMRI likea mass with round or oval shape, smooth margin, center high signal intensity on T2 weighted images.

\section{References}

1. Jenkins S, Kachur ME, Rechache K, Wells JM, Lipkowitz S (2021).Rare Breast Cancer Subtypes. Current Oncology Reports, 23: 54.

2. Boisserie-Lacroix $\mathbf{M}$, HurteventLabrot G, Ferron S, Lippa N, Bonnefoi H, Mac Grogan G (2013). Correlation between imaging and molecular classification of breast cancers. Diagnostic and Interventional Imaging, 94(11):1069-1080.

3. Ng CKY, Schultheis AM., Bidard FC, Weigelt B, Reis-Filho JS (2015). Breast cancer genomics from microarrays to massively parallel sequencing: Paradigms and new insights. Journal of the National Cancer Institute, 107 (5): 113.

4. Fan M, Li H, Wang S, Zheng B, Zhang J, Li L (2017). Radiomic analysis reveals DCE-MRI features for prediction of molecular subtypes of breast cancer. PLOS ONE, 12 (2): 1-15.

5. Allarakha A, Gao Y, Jiang H, Wang PJ (2019).Prediction and prognosis of biologically aggressive breast cancers by the combination of DWI/DCE-MRI and immunohistochemical tumor markers.Discovery Medicine, 27 (146):7-15.

6. Osman NM, Nivinei C, AbdRaboh NM (2014). Triple negative breast cancer: MRI features in comparison to other breast cancer subtypes with correlation to prognostic pathologic factors. The Egyptian Journal of Radiology and Nuclear medicine, 45: 1309-1316.

7. Issar $\mathbf{P}$, Sinha $\mathbf{S}$, Ravindranath $\mathbf{M}$, Issar SK (2020).MRI Features of Different Molecular subtypes of Breast Cancer.Indian Journal ofApplied Radiology, 6 (1): 151.

8. Schrading S, Kuhl CK (2008).Mammographic, US and MR 
imaging phenotypes of familial breast cancer. Radiology; 246:58-70.

9. Navarro Vilar L, Alandete Germán SP, Medina García R, Blanc García E, Camarasa Lillo N, Vilar Samper J (2017).MR ImagingFindings in Molecular Subtypes of Breast Cancer According to BIRADS System. The Breast Journal, 23 (4):421-428.

10. Uematsu T, Kasami M, Yuen S (2009). Triple-Negative breast cancer: correlation between MR imaging and pathologic findings. Radiology, 250 (3), 638-647.

11. Youk JH, Son EJ, Chung J, Kim JA, Kim EK (2012).Triple - negative invasive breast cancer on dynamic contrast enhanced and diffusion weighted MR imaging comparison with other breast cancer subtypes.European Radiology, 22 (8): 1724-1734.

12. Grimm LJ, Johnson KS, Marcom PK, Baker JA, Mary SS (2014). Can breast cancer molecular subtype help to select patient for preoperative MR Imaging. Radiology,274(2):352-358.

13. Azzam, H, Kamal, R, El-Assaly H, Metwally LIA (2019). The value of dynamic contrast-enhanced MRI in differentiating triple-negative breast cancer from other subtypes. Egyptian Journal of Radiology and Nuclear Medicine, 50 (1):106

14. Sung JS, Jochelson MS, Breman S, Joo S, Wen YH (2013).MR Imaging features of triple negative breast cancer.The Breast Journal, 19: 643-649.

15. Surov A, Chang YW, Li L, Martincich L, Partridge SC, Kim JY, Wienke A (2019). Apparent diffusion coefficient cannot predict molecular subtype and lymph node metastases in invasive breast cancer: A multicenter analysis.
BioMed Central (BMC) Cancer, 19 (1): 1043 . 